\title{
RELATIVE MODULATION SENSITIVITIES OF THE RED AND GREEN COLOR MECHANISMS
}

\author{
Carol M. Cicerone and Dantel G. Green \\ Vision Research Laboratory. University of Michigan, Ann Arbor. MI 48109. U.S.A.
}

(Received 29 March 1977)

\begin{abstract}
The sensitivities of the human green and red cone mechanisms to sinusiodally flickering light were found to be near equal and independent of field size and estimation method. No wavelength dependency of modulation sensitivity was found. An observer who lacks the red cone pigment (protanope) did not show unusually high flicker sensitivity to green light of $525 \mathrm{~nm}$. The modulation transfer function for the normal observer measured with a green flickering test on a red background is identical to that on a green background when the backgrounds have been equated for the green cones by the protanope. The same is true for the contrast transfer function of the normal observer when determined with a green grating on the red and green backgrounds equated by the protanope.
\end{abstract}

\section{INTRODUCTION}

Several years ago, one of us reported data on the spatial and temporal transfer functions of the three color mechanisms of human trichromatic vision (Green, 1968, 1969). More recently, Kelly $(1973,1974)$ has published the results of a similar series of experiments, but with some strikingly different conclusions. In contrast to Green's finding that the transfer functions of the red and green mechanisms are nearly identical in peak sensitivity. Kelly reports that. under some conditions. the green mechanism peak is more sensitive by a factor of $5-8$ than the red. Supersensitivity of the green mechanism is not apparent when viewing chromatic gratings under ordinary circumstances, but appears only after the red mechanism has been depressed by suitable chromatic adaptation. These findings have important theoretical implications. They cannot be explained by assuming that the well-established red-green opponent interactions reduce normal sensitivity. Red-green opponent color interactions are known to be linear or approximately linear functions of the photo-pigment quantum-catch functions (Larimer, Krantz and Cicerone, 1974). Such linear processes will not suffice to explain supersensitivity of the green mechanisms as well as De Lange's (1958) finding that peak modulator sensitivity is nearly independent of wavelength. To appreciate this. consider what happens with stimuli in the middle and long wave portions of the spectrum. These stimuli will affect the green and red cones to varying extents. Linear differences between signals from a supersensitive green mechanism and a less sensitive red mechanism cannot account for a constant and depressed sensitivity at all wavelengths. If at any particular wavelength one assumes a suitable weighting to compensate for the unequal sensitivities of the underlying systems, then at shorter wavelengths one expects relatively entranced sensitivity and, at longer wavelength, relatively depressed sensitivity. What seems to be required if Kelly's observations are correct is a strong nonlinear interaction between the outputs from two different kinds of cone systems.

Why these interactions are present under the condi- tions of some experiments and not those of Green's or those of the several recent independent determinations of the temporal and spatial transfer functions of the color mechanisms (Estévez and Spekreijse. 1974; Cavonius and Estévez, 1975a, b; Estévez and Cavonius, 1975: Cicerone. 1974) has been an unanswered puzzle. Estévez and Cavonius (1976) and Kelly (1976) give a detailed discussion of some of these issues. Several procedural differences might be important. Green used small targets with sharp edges. superimposed on a larger. uniform adapting field. and Kelly used a large $\left(8^{\circ}\right)$ circular test field which coincided with the adapting field. To estimate the sensitivity of the color mechanisms, Green (1968, 1969) varied the test field radiance and Kelly (1973, 1974) varied the adapting radiance.

In this paper, we first report our attempts to identify which procedural differences lead to the discrepancies between different determinations of the temporal transfer functions of the red and green cone systems. Disconcertingly, none of the above-mentioned procedural differences seem to account for the differences in findings for the red and green mechanisms. Using a large test field and adjustment of adapting field radiance to determine sensitivity, we fail to find supersensitivity of the green mechanism; instead. we replicate Green and the other workers in showing the near equality of the isolated red and green mechanism peak modulation sensitivity. Three other experiments were designed in pursuit of green supersensitivity, but only served to cast further doubt on its existence.

\section{METHODS}

The experiments of Figs 1, 2. 3, and 4 were conducted in an apparatus similar to that described by Green. A flickering test field $5.0^{\circ}$ diameter was formed by imaging the light from an electronically modulated Sylvania CoolWhite fluorescent tube on a $25 \mathrm{~mm}$ artificial pupil. The color and intensity of the test field was varied by means of neutral filters (Oriel) and narrow band interference filters (Baird Atomic). The mean irradiance and depth of modulation was measured at each wavelength with a calibrated pin-10 photodiode (UDT) and suitable infrared 
Table 1. The estimated true modulation thresholds ( \pm S.E.M.) for the red and cone mechanisms measured with $10 \mathrm{~Hz}$ sinusoidally flickering light.

\begin{tabular}{cccccc}
\hline & \multicolumn{2}{c}{ Red/Blue-Green } & \multicolumn{2}{c}{ Green/Purple } \\
\hline & $2^{2}$ & $8^{\circ}$ & $2^{\circ}$ & $3^{\circ}$ \\
$m_{\tau}^{*}$ & $1.19 \pm 0.61$ & $1.22 \pm 0.52$ & $1.62 \pm 0.91$ & $0.98 \pm 0.41$ \\
$m_{\mathrm{B}}^{*}$ & $1.22 \pm 0.52$ & $1.19 \pm 0.48$ & $2.50 \pm 0.62$ & $1.73 \pm 0.46$ \\
\hline
\end{tabular}

Small $\left(2^{\circ}\right)$ or large $\left(8^{\circ}\right)$ tests on mechanism isolating backgrounds are shown. Thresholds were estimated by varying test luminance, $m_{T}^{*}$, or by varying background luminance, $m_{\mathrm{B}}^{*}$. The details of the two estimation methods are given in the Methods section.

blocking filters. The CIE photopic sensitivity curve was used to determine the supplemental filters necessary at each wavelength to keep luminance constant. The retinal illuminance was determined at $i=575 \mathrm{~nm}$ using the method described by Westheimer (1966). The subjects fixated the flickering field through the artificial pupil and adjusted a ten-turn potentiometer controlling the modulation until satisfied that the flicker could be detected. A second channel. derived from a tungsten source. provided a steady $12^{2}$ diameter color adapting background. The background was either green $(i=520)$ or red $(j=625)$ in color. The intensity of the background could be adjusted by rotating a $2 \log$ unit neutral density wedge (Kodak). In some experiments. the flickering field was replaced by a $4^{\circ}$ (long) $\times 8^{\circ}$ (wide) field of vertical stripes. The sinusoidal gratings were generated on a CRT having a P31 phosphor, and were viewed through a broad-band interference filter with $50 \mathrm{~nm}$ half-band width centered at $500 \mathrm{~nm}$. The resulting field of stripes was green in color with approximately the same hue and saturation as the $520 \mathrm{~nm}$ background.

The experiment of Table 1 was conducted on a threechannel Maxwellian view apparatus. The test stimulus was formed by combining two channels-one steady and the other sinusoidally flickering. The flicker was produced with an arrangement of rotating polarizers. Flickering stimuli could be varied in depth of modulation, keeping the mean luminance fixed by means of calibrated Wratten neutral density filters. The third channel provided steady adapting backgrounds. The Maxwellian view lens formed a circular image, $1 \mathrm{~mm}$ in diameter, which entered the observer's eye through the natural pupil. The background was an $8^{\circ}$ field. The superimposed test was either an $8^{\circ}$ field or a concentric $2^{\circ}$ field. The subject's head was fixed with a bite bar. The sinusoidally flickering component of the test field was presented to the observer by means of an electromagnetic shutter (Uniblitz, Vincent Associates).

An up-and-down staircase procedure was used (Cornsweet, 1962) to determine the flicker fusion threshold. Half the trials were started well below threshold, the other half well above. The experimenter varied percent modulation in a sequence of steps. The subject responded "yes" if he saw flicker and "no" if he did not. After "yes" responses, modulation was decreased and after "no" responses, it was increased. Two transition points were used in each series of trials. By pooling several day's results, it was possible to plot frequency-of-seeing functions. Smooth curves were fit by eye and the threshold modulation corresponding to $50 \%$ frequency-of-seeing was estimated.

\section{Methods of estimating the true modulation sensitivities}

The temporal variation of the quantum catch in any mechanism for a sinusoidally flickering light of irradiance $E_{1}$ and wavelength $\lambda_{1}$ on a steady background, irradiance $E_{2}$, wavelength $\lambda_{2}$, may be written

$$
Q(t)=E_{1} \rho\left(\lambda_{1}\right)(1+m \sin 2 \pi f t)+E_{2} \rho\left(\lambda_{2}\right)
$$

where $\rho(\lambda)$ is the spectral absorption curve for that mechanism. This equation expresses that the background as well as the test affects the mechanism of interest. The true modulation of the mechanism then is not $m$, but

$$
m^{*}=m E_{1} \rho\left(i_{1}\right)\left[E_{1} \rho\left(i_{1}\right)+E_{2} \rho\left(i_{2}\right)\right] .
$$

To estimate the true modulation one can manipulate either the test irradiance, $E_{1}$. by a factor of $k_{\mathrm{T}}$ or the background irradiance. $E_{2}$. by a factor of $k_{\mathrm{B}}$. For $E_{2}$ large compared to $E_{1}$ and moderate values of $k_{\mathrm{T}}$, the factor increase in $E_{1}$. Green (1969) noted and experimentally confirmed the approximation

$$
m_{\mathrm{T}}^{*}=m_{\mathrm{t}} /(1+\eta)=m_{2} /\left(1+\eta / k_{\mathrm{T}}\right)
$$

where $m_{l}$ is the measured modulation threshold for test irradiance $E_{1}, m_{2}$ is the threshold for test irradiance $k_{\mathrm{T}} E_{1}$ and $\eta=E_{2} \rho\left(\lambda_{2}\right) / E_{1} \rho\left(\lambda_{1}\right)$. Solving for $\eta$, Green computed true modulation from his measured values according to the formula

$$
m_{\mathrm{T}}^{*}=\left(k_{\mathrm{T}} m_{2}-m_{1}\right) /\left(k_{\mathrm{T}}-1\right) .
$$

Instead of manipulating test intensity, Kelly (1973) manipulated the background intensity by factors $k_{\mathrm{g}}$. Kelly noted and experimentally tested the following:

$$
m_{1}^{\prime} /(1+\eta)=m_{2}^{\prime} /\left(1+k_{B} \eta\right)
$$

where $m_{1}^{\prime}$ is measured on the dimmer background, $m_{2}^{\prime}$ on the brighter and $r=m_{2}^{\prime} / m_{1}^{\prime}$. His correction rule for $k_{B}>1$ was thus:

$$
\begin{aligned}
& m_{\mathrm{B}}^{*}=m_{1} /\left[k_{\mathrm{B}}-1\right) /\left(k_{\mathrm{B}}-r\right) \\
&=\left(k_{\mathrm{B}} m_{1}^{\prime}-m_{2}^{\prime}\right) /\left(k_{\mathrm{B}}-1\right) .
\end{aligned}
$$

\section{RESULTS}

\section{Are procedural differences important?}

The first experiment was directed at determining the modulation sensitivity of the red and green colour mechanisms using a more precise psychophysical procedure than that employed previously (see Methods). The green mechanism was isolated using green flicker (Wratten 45 and 58 , in combination) on a bright purple background (Wratten 35), and the red mechanism was isolated using red flicker (Wratten 72B) on a bright blue-green background (Wratten 75). The adapting background in isolating the chosen cone mechanism depresses its sensitivity by an unknown amount. This amount was estimated by either varying the test luminance (method used by Green, 1969) or the background luminance (method used by Kelly, 1973), and using the resulting change in modulation thresholds to adjust for true sensitivity. In this set of experiments, we used a green test and a purple background to isolate the green mechanism, as, did Kelly $(1973,1974)$ and Green (1969). For the original 
experiments of Green, the rationale behind using a fxed purple background had been to depress the sensitivity of the blue as well as the red mechanisms. Varying the green test stimulus presented no problem. However, attenuating the purple background by a known amount can be difficult since most neutral filters are not fiat. Kodak Wratten neutral density filters. for example. are denser in the blue than in the red part of the spectrum. At long wavelengths, for example, a calibration based on the CIE photopic sensitivity would tend to underestimate the effective density of the purple background, as seen by the green cones. On the other hand, contributions to the CIE from blue cones might tend to cause errors of the opposite kind. Nonetheless, we used a purple background and CIE-based calculations. The tests were flickered at $10 \mathrm{~Hz}$, which is near the peak of the red and green mechanisms' modulation transfer function (Green, 1969). At lower frequencies, Green reported that the red system was slightly more sensitive than the green and at high frequencies the reverse was true. These differences were quite small compared to the red-green differences reported by Kelly. The red and green test luminances were 1.6 and 1.4 $\log t d$. respectively. The blue-green background luminance (comparable to Kelly's) was $2.9 \mathrm{log}$ td. The purple background luminance $(3.31 \log t d)$ was chosen so that the modulation sensitivity measured upon it with the green test matched that for the red test on the blue-green background. Measurements were also made with test or background luminance dimmed by approx. $0.5 \log$ unit. Table 1 shows the results for a single subject. No matter whether sensitivities $m_{\mathrm{T}}^{*}$ and $m_{\mathrm{B}}^{*}$ were estimated using the procedure described by Green (1969) or that used by Kelly (1973). respectively, the red and green cone mechanisms had equal sensitivity. This was true whether or not the test was the same size as the background $\left(8^{\circ}\right)$, or smaller than it $\left(2^{\circ}\right)$. Furthermore, the green thechanism was not supersensitive under any condition.

\section{Wavelength dependence of modulation sensitivity}

In the trichromatic theory of color vision. the appearance of spectral lights varies as one moves across the spectrum because of variation in the contributions of the three types of cones. If one of these receptors had an unusually high flicker sensitivity, then an inctease in sensitivity might be expected in those parts of the spectrum which stimulate it most strongly. The next experiment was designed to examine this possibility by determining the dependence of modulation sensitivity on wavelength. The colored stimuli were of equal luminance ( $40 \mathrm{td}$ ), and flickered at a frequency of $10 \mathrm{~Hz}$, which is close to the peak in the modulation transfer function (De Lange, 1954; Kelly, 1961; Green, 1969). Results from two observers are shown in Fig. 1 (closed symbols). Threshold modulation is plotted on an expanded logarithmic scale and wavelength is plotted on a linear scale. The findings show that at 40 td modulation sensitivity is nearly independent of wavelength, over the range of wavelengths tested. To be certain that a small error in equating the luminances was not obscuring an interesting wavelength dependency, the measurements were repeated at $400 \mathrm{td}$. Both observers showed a slight improvement at all measured wavelengths (open symbols, Fig. 1), but no wavelength dependency.

\section{Protanope's sensitivity to green flicker}

We do not find supersensitivity when a green flickering light is viewed without an adapting background. One might hypothesize that this results from a redgreen antagonism because Kelly (1973) reports super-

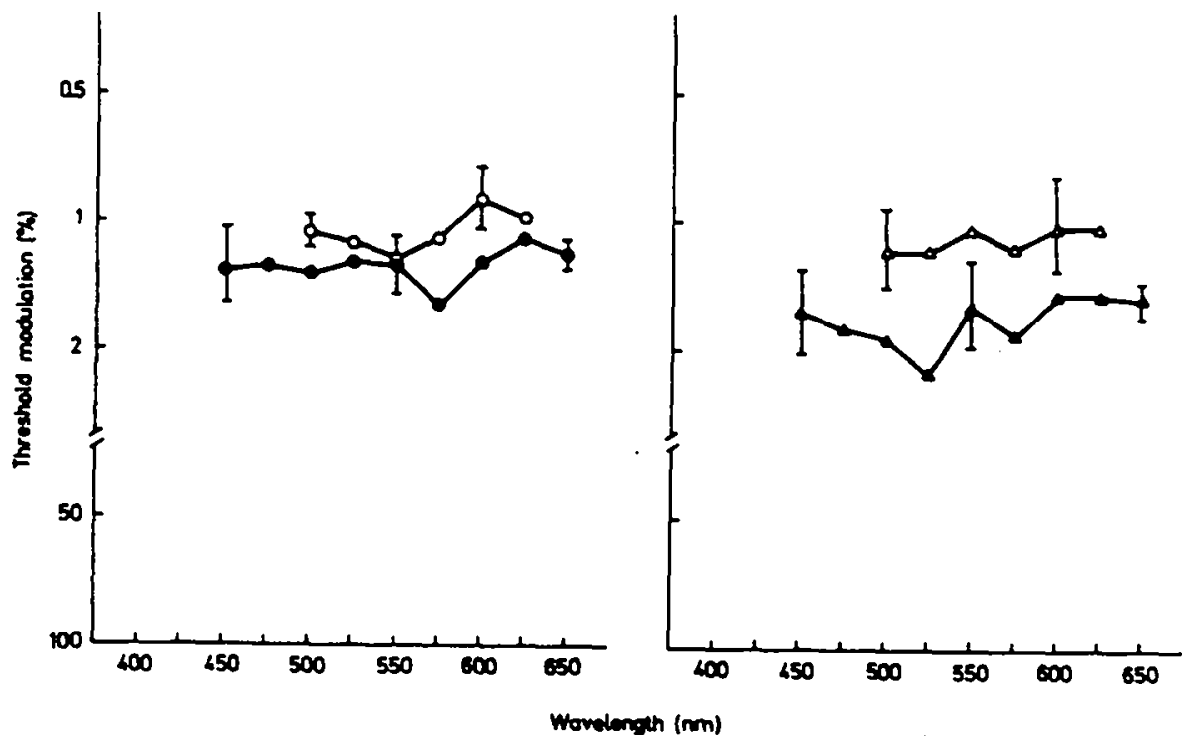

Fig. 1. The sensitivity of normal observers to $10 \mathrm{~Hz}$ sinusoidal flicker was measured using monochromatic lights of equal luminance. The circles show the results for subject DGG and the triangles for subject CMC. The open symbols are the results at 400 td; the closed symbols are the results at 40 td. Each point is the average of eight measurements. The error bars at selected points mark \pm 1 standard deviation. No wavelength dependence was found. 


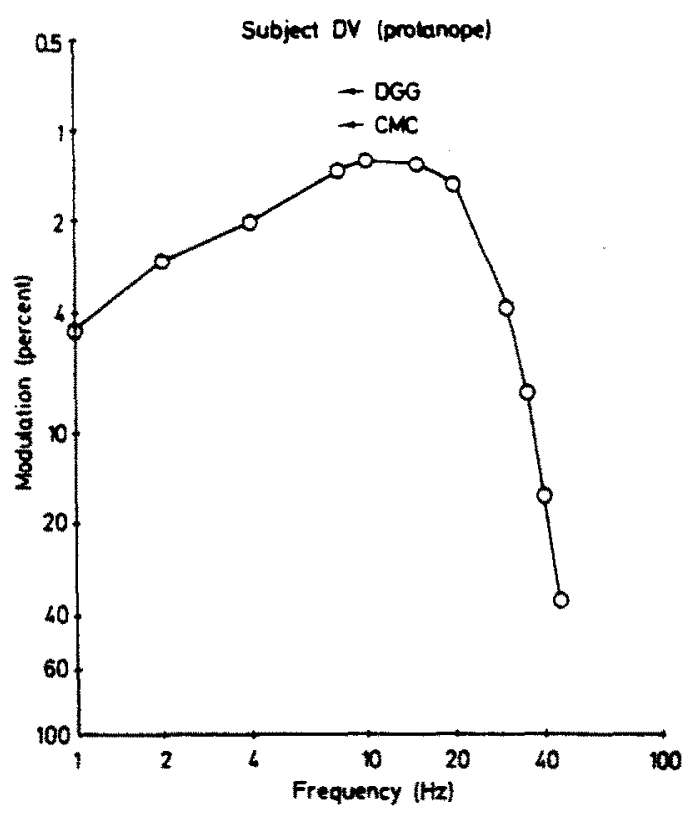

Fig. 2. The modulation transfer function of a protanope was measured using green $(525 \mathrm{~nm})$ flicker. Data points are marked by the open circles. Each point is the average of four measurements made at a mean retinal illumination of $300 \mathrm{td}$. Arrows mark the sensitivity at $8 \mathrm{~Hz}$ for two normal observers. The protanope does not show unusual sensitivity to green flicker.

sensitivity only when green gratings are viewed on a mechanism-isolating purple background. Since the purple background affects the green mechanism as well, the sensitivity of the green system must be deduced by varying the background or the test intensity. While there is nothing to indicate that the estimating procedures are faulty, a direct measure would obviously be better.
It is generally agreed that the color defective called the protanope lacks the normal red cone pigment and that his failure to distinguish colors in the middle and long wavelength part of the spectrum is due to the fact that only the green pigment is excited by these wavelengths (Rushton. 1963; Wald. 1966). Therefore, one might expect that if the normal modulation sensitivity for green stimuli is depressed by antagonism from the red cones, then the protanope will be supersensitive. To test this. the flicker sensitivity of a protanope for the green $(\lambda=525)$ was measured without an adapting background. The results are shown in Fig. 2. The arrows indicate the sensitivity of the two normals at $8 \mathrm{~Hz}$ under the same viewing conditions. The protanope is not more sensitive than normal.

\section{Is the normal green inechanism supersensitive?}

The significance of the previous result can be questioned since it is the sensitivity of the green mechanism in the normal, not the color defective, that is of interest. There seems to be general agreement that, without a background, normal observers do not show unusual sensitivity to green flicker or gratings (see Figs. 1 and 2. for example; also De Lange. 1958; Kelly, 1973). What is in question is whether normals are supersensitive by a factor of 5-8 when the red mechanism is depressed by a red adapting background. The answer can be directly determined by using the protanope.

Since the protanope lacks the red cone pigment, we can used him to equalize the effect of a red background and a green background on the green pigment. Figure 3 shows the protanope ficker increment threshold curves for the same green $(525 \mathrm{~nm}) .8 \mathrm{~Hz}$ flickering light against a green $(520 \mathrm{~nm})$ or against a red $(625 \mathrm{~nm})$ background. The curves were generated by setting the mean luminance of the $8 \mathrm{~Hz}$ flick-

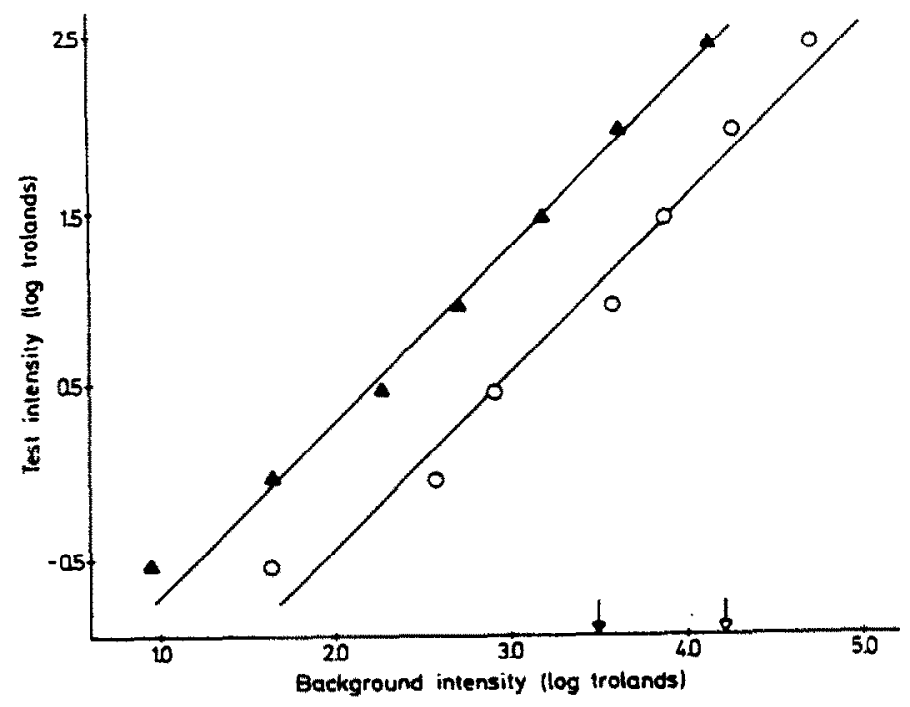

Fig. 3. The protanope was used to equate the effectiveness of a red $(625 \mathrm{~nm}$, open circles) background and a green ( $520 \mathrm{~nm}$. closed triangles) background for the green pigment. For any given mean intensity of the green $(525 \mathrm{~nm}), 8 \mathrm{~Hz}$ test light flickered at $100 \%$ modulation, the protanope adjusted the background so the flicker was just at threshold. Each point is the average of four measurements. The straight lines have a slope of unity and show the Weber's law behavior of the data. Arrows mark the background intensity in trolands chosen for the experiment of Fig. 4, the open arrow for the red background and the closed arrow for the green background. 


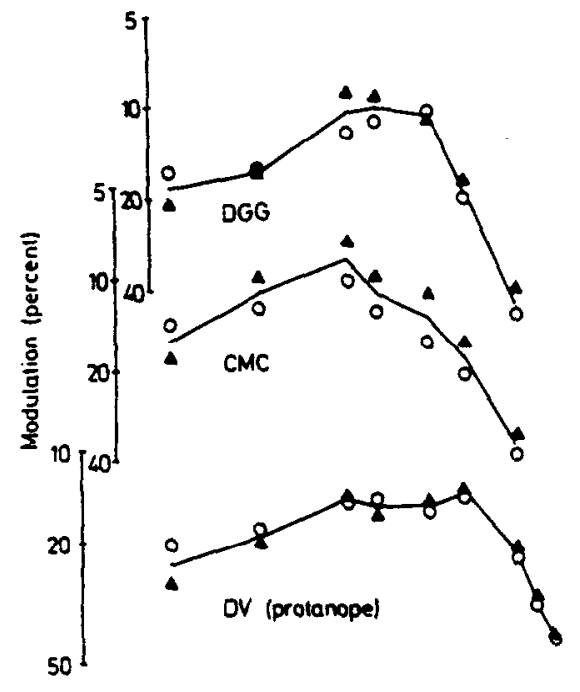

$\begin{array}{llllll}1 & 20 & 20 & 40 & 100\end{array}$

Fig. 4. Modulation sensitivity was measured for two normals and a protanope for a green $(525 \mathrm{~nm})$ test on a red $(625 \mathrm{~nm}$. open circles) and a green $(520 \mathrm{~nm}$, closed triangles) background. The background intensities had previously been equated by the protanope at $8 \mathrm{~Hz}, 100 \%$ modulated flicker. The measured sensitivities were nearly identical for all observers whether a red or a green background was used.

ering light. modulated at $100 \%$, then asking the protanope to adjust the adapting background so that the flicker is just visible. The functions follow the familiar Weber's law and their separation along the abscissa gives the brightness factor we need to equate these two backgrounds for the green cones.

Now, two normal observers and the protanope viewed the same green flickering test at full intensity against either the equated red or green background. The backgrounds were selected so that the green flickering light was $0.5 \log$ units above threshold. The results in Fig. 4 for the protanope are identical for either background and further verifies that for all frequencies the backgrounds were equated for this observer. Since the backgrounds have been equated for the green cones, even though they strongly depress sensitivity, no calculations are required to adjust for their relative effect on the green cone mechanism. If supersensitivity exists, then, since the red background is effective in desensitizing red cones, normals should be more sensitive to green on red than to green on green. Figure 4 shows that this does not happen. Instead, the normal is slightly less sensitive to green on red than to green on green.

The same is true if the temporally modulated target is replaced by a spatially modulated sinusoidal grating. Figure 5 shows the contrast sensitivity of two normals and a protanope measured with a $4^{\circ} \times 8^{\circ}$ field superimposed on the protanopically equated red and green backgrounds.

\section{Discussion}

The data of Table 1 fail to demonstrate a difference

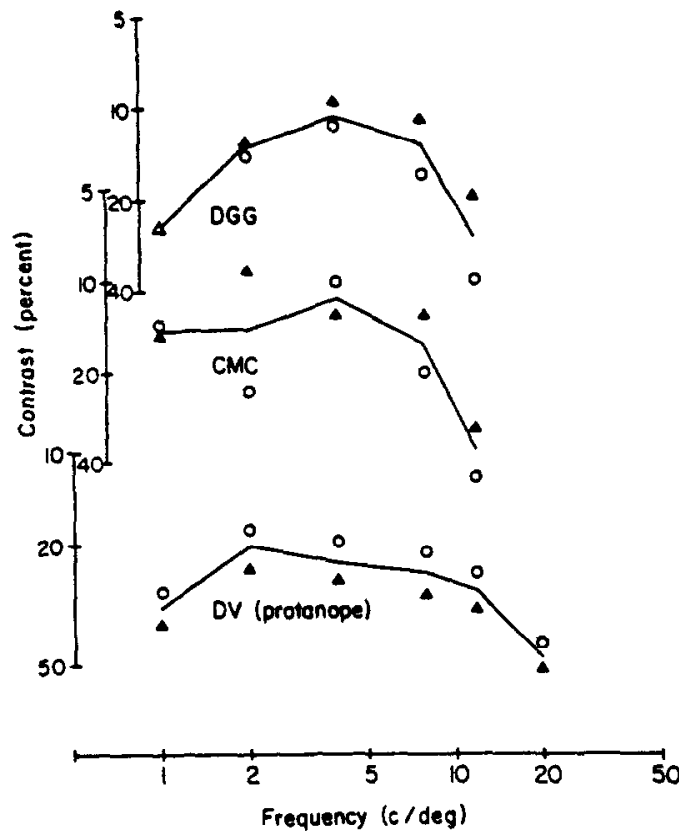

Fig. 5. Contrast sensitivity for two normals and a protanope for a green grating on a red $(625 \mathrm{~nm}$, open circles) and a green ( $520 \mathrm{~nm}$. closed triangles) background. The relative intensities of the backgrounds were the same as in Fig. 4. The contrast sensitivities for a green grating/ green background and for a green grating/red background were nearly identical for all observers.

between modulation thresholds with green/purple and red/blue-green test/adaptation conditions. It would be a mistake, however, to interpret such data as proving that the flicker sensitivities of the red and green cone mechanisms are exactly identical. First, the measurements in the table are for one temporal frequency $(10 \mathrm{~Hz})$. In fact, $10 \mathrm{~Hz}$ was selected since it is the frequency where Green (1969) found the red and green systems had equal sensitivities. At other frequencies, Green reported differences which he interpreted as indicating that the red and green mechanisms had different dynamic characteristics. Second, the formulae used to estimate the threshold modulation produced in individual red and green cones involve taking a difference between experimentally determined quantities which are not very different in magnitude. To be more precise, let us consider the case where modulation is estimated by varying test luminance. The true modulation. $m_{\mathrm{T}}^{*}$, is given by Eqn 6. Specifically, the last entry on the right in Table 1 was computed from $m_{2}^{\prime}=5.11 \pm 0.31, m_{1}^{\prime}=2.75 \pm$ 0.23 . and $k_{\mathrm{B}}=3.31$. Using the usual \pm S.E. of the mean. the threshold modulations could lie within a factor of $1 \pm 0.06$ of the mean. However, the true value of $m_{B}^{*}$ could lie anywhere within a factor of $1 \pm 0.27$ of the mean. While our results clearly cannot exclude the possibility that the sensitivities of the red and green differ, they do show that if such differences exist, they must be rather small.

Our data do not show supersensitivity. The experiment of Fig. 2 is a good test of supersensitivity only if the protanope lacks the red cones as well as the normal red cone pigment. However. if the protanope 
has both the normal set of green cones and "red" cones, each set filled with chlorolabe, then our test on the protanope is obviated. In this case, the neuronal connections from red cones to green cones could mimic the normal antagonism. Since both sets are filled with the same pigment, there would be no way of selectively depressing the "red" cones.

The results of Fig. 4 cannot be attacked on this ground, for they rest on the well-established fact that protanopes do not have the normal long wavelength (red) pigment. Neither Fig. 4 nor Fig. 5 shows evidence for supersensitivity. In fact, if anything, the opposite is true. On red and green backgrounds equated by the protanope, it is slightly more difficult for the normal observer to detect the green flicker and gratings against the red background than the green background. Could the failure to find supersensitivity stem from the pigment in the green cones of the normal observer being different from the protanope's long wavelength pigment? (Alpern and Moeller, 1977; Bastian, 1976.) To answer this. we need to consider how the absorption spectrum of the protanope green cone pigment affects the results of our two color experiments. If the steady background is significantly brighter than the steady component of the flickering light. Eqn 2 reduces to

$$
m_{\tau}^{*}=\frac{m E_{1} \rho\left(\lambda_{1}\right)}{E_{2} \rho\left(\lambda_{2}\right)}
$$

and we see the modulation is inversely proportional to the green cone absorption at $\lambda_{2}$. Now we have used the protanope to equate the contribution of the steady background $E_{2} \rho\left(\lambda_{2}\right)$ for $\lambda_{2}=520 \mathrm{~nm}$ and $i_{2}=625 \mathrm{~nm}$. The true modulation for the red background will be 5-8 times lower than we measure only if a dimmer background is used. The ratio of absorbance at $520 \mathrm{~nm}$ must be 5-8 times larger for the normal's green concs than for the protanope's. This is not possible since the green cones' absorption spectra should be at least as narrow as the normal photopic sensitivity curve, and normals are only three to four times relatively more sensitive to $625 \mathrm{~nm}$ than protanopes (Wald, 1966).

Acknowledgements-We thank Patricia Hatfield for expertly preparing the drawings and typing the manuscript. This research was supported by grant EY 00379 to D.G.G. and postdoctoral fellowship EY 02161 to C.M.C. from the National Eye Institute, U.S. Public Health Service.

\section{REFERENCES}

Alpern M. and Moeller J. (1977) The red and green cone pigments of deuteranomalous trichromacy. $J$. Phisiol 266. $647-675$.

Bastian B. L. (1976) Individual differences among the photopigments of protan observers. Ph.D. dissertation. University of Michigan.

Cavonius C. R. and Estévez O. (1975a) Contrast sensitivity of individual colour mechanisms of human vision. $J$. Physiol. 248, 649-662.

Cavonius C. R. and Estevez O. (1975b) Sensitivity of human color mechanisms to gratings and flicker. J. opt. Soc. Am. 65, 966-968.

Cornsweet T. N. (1962) The staircase-method in psychophysics. Am. J. Psychol. 75, 485-491.

Cicerone C. M. (1974) Theory of luminance detection and measurement of the impulse responses and frequency responses of color mechanisms of the eye. Ph.D. dissertation. University of Michigan.

De Lange H. (1954) Relationship between critical hickerfrequency and a set of low-frequency characteristics of the eye. J. opt. Soc. Am. 44. 380-389.

De Lange H. (1958) Research into the dynamic nature of the human fovea-cortex systems with intermittent and modulated light. $J$. opt. Soc. Am. 48, 777-789.

Estévez O. and Spekreijse H. (1974) A spectral compensation method for determining the flicker characteristics of the human colour mechanisms. Vision Res. 14, 823-830.

Estévez O. and Cavonius C. R. (1975) Flicker sensitivity of the human red and green color mechanisms. Vision Res. 15, 879-881.

Estévez O. and Cavonius C. R. (1976) Modulation sensitivity of human color mechanisms. J. opt. Soc. Am. 66, 1436-1438.

Green D. G. (1968) The contrast sensitivity of the colour mechanisms of the human eye. J. Physiol. 196, 415-429.

Green D. G. (1969) Sinusoidal flicker characteristics of the color-sensitive mechanisms of the eye. Vision Res. 9. $591-601$.

Kelly D. H. (1961) Visual responses to time-dependent stimuli. I. Amplitude sensitivity measurement. $J$. opt. Soc. Am. 51, 422-429.

Kelly D. H. (1973) Lateral inhibition in human colour mechanisms. J. Physiol. 228, 55-72.

Kelly D. H. (1974) Spatio-temporal frequency characteristics of color-vision mechanisms. J. opt. Soc. Am. 64, 983-990.

Kelly D. H. (1976) Differences between red and green color-mechanism characters: Interaction or artifact? $J$. opt. Soc. Am. 66, 1430-1435.

Larimer J., Krantz D. H. and Cicerone C. M. (1974) Opponent-process additivity. I: Red/green equilibria. Vision Res. 14, 1127-1140.

Rushton W. A. H. (1963) A cone pigment in the protanope. J. Physiol. 168, 345-359.

Wald $G$. (1966) Defective color vision and its inheritance. Proc. Natn. Acad. Sci., U.S.A. 55. 1347-1363.

Westheimer G. (1966) The Maxwellian view. Vision Res. 6, 669-682. 